\title{
Comparing Horizontal and Vertical Surfaces for a Collaborative Design Task
}

\author{
Brianna Potvin, Colin Swindells, Melanie Tory, and Margaret-Anne Storey \\ Department of Computer Science, University of Victoria, Engineering/Computer Science Building (ECS), \\ Room 504, P.O. Box 3055, STN CSC, Victoria, BC, Canada V8W 3P6 \\ Correspondence should be addressed to Melanie Tory, mtory@cs.uvic.ca
}

Received 13 October 2011; Revised 20 January 2012; Accepted 26 January 2012

Academic Editor: Antonio Krüger

Copyright (c) 2012 Brianna Potvin et al. This is an open access article distributed under the Creative Commons Attribution License, which permits unrestricted use, distribution, and reproduction in any medium, provided the original work is properly cited.

We investigate the use of different surface orientations for collaborative design tasks. Specifically, we compare horizontal and vertical surface orientations used by dyads performing a collaborative design task while standing. We investigate how the display orientation influences group participation including face-to-face contact, total discussion, and equality of physical and verbal participation among participants. Our results suggest that vertical displays better support face-to-face contact whereas side-byside arrangements encourage more discussion. However, display orientation has little impact on equality of verbal and physical participation, and users do not consistently prefer one orientation over the other. Based on our findings, we suggest that further investigation into the differences between horizontal and vertical orientations is warranted.

\section{Introduction}

Although considerable effort has been devoted to the design of tabletop interfaces, the tasks and situations for which they are preferable to vertical displays are less certain. Marshall et al. [1] argue that there is a need for more studies investigating how interfaces encourage or inhibit group participation. One aspect worthy of consideration is orientation: horizontal versus vertical. While vertical displays are known to be effective for presentations [2], some researchers have argued that horizontal displays might support more seamless interactions among small groups for collaborative tasks [2,3]. Consequently, substantial effort has been placed on designing effective interfaces and applications for tabletop displays.

There are surprisingly few studies that explicitly compare how horizontal and vertical displays influence collaborative work. The studies that do make this comparison are highly varied in terms of their configuration (task, number of users, input, etc.), making it difficult to draw conclusions for any particular configuration. As researchers working with both horizontal and vertical displays, we were curious about how the display orientation influences group participation. We focus particularly on dyads (pairs of participants) working on a software design task. This is a very common task in software engineering and is often performed by pairs or small groups [4]. Our choice of task was also motivated by current interest in building digital tools to support earlystage software design [5-7]; however, the results may be relevant to dyads performing other types of open-ended tasks on shared displays.

By examining the differences between horizontal and vertical displays, we expect to influence groupware design and the choice of display orientation for particular tasks. At the outset of our study, we expected to observe benefits for horizontal displays in terms of group participation, but surprisingly, we did not. Our results indicate that vertical displays may encourage more face-to-face contact among dyads (i.e., they glance at each other's faces more often), and side-by-side arrangements of people may encourage more discussion, at least when users are standing, which was the configuration used in our study.

In the next section, we describe previous studies and their findings and relate these to our experimental design. We then describe our study objectives and hypotheses (Section 3), our methods (Section 4), followed by results (Section 5), discussion (Section 6), and an outlook to future work (Section 7). 


\section{Related Work}

2.1. Shared Displays. Design considerations for shared displays have been studied extensively. Scott et al. [3] provide an overview of design suggestions specific to horizontal displays. In this section, we summarize some of the key findings from this body of research.

Observations of groups working together on shared displays have revealed that people frequently switch between loosely and closely coupled styles of work [8]. Research shows that even during loosely coupled work, maintaining awareness (e.g., what is being worked on by whom, and how your actions will affect others) is critical to ensure efficient and effective team coordination [9]. Supporting awareness is even more critical when team members are working at different locations. Another important factor for many applications is territoriality. Distinguishing between personal and shared work territories mimics the way people work naturally and supports transitions between individual and shared work [10]. The positioning of users relative to each other and to the display also impacts collaboration. For example, Hawkey et al. [11] found that collaboration was perceived to be more effective and more enjoyable when two users were both positioned close to a large display. Also, numerous papers have considered technological challenges and design considerations for multidisplay environments (e.g., [12-14]).

Studies of how people use whiteboards and other large nondigital drawing surfaces are also relevant. People's interactions with such surfaces have been studied in many different contexts, but these studies have usually not considered the effect of the surface orientation. For example, Walny et al. characterized the visual constructs that people use on whiteboards and derived design implications for information visualization tools. Tang [15] studied interactions with shared drawing surfaces for a group design task but focused on categorizing people's physical actions in the drawing space and the function of those actions. Tang et al. [16] examined how whiteboards facilitate transitions between tasks and different modes of activity, particularly for asynchronous group work. Perhaps most relevant to our study, Damm et al. [6] conducted two field studies examining how software developers use whiteboards to design software using UML (Unified Modeling Language). They found that approximately $80 \%$ of the drawings were formal UML diagrams and the remaining $20 \%$ were informal or incomplete drawings. They also made several specific recommendations for the design of interactive UML diagramming tools. While these and other whiteboard studies contribute to our understanding of how people use surfaces collaboratively, they do not directly address the differences between horizontal and vertical displays.

\subsection{Studies Comparing Horizontal and Vertical Displays. Few} studies have focused on explicitly comparing horizontal and vertical displays. Table 1 situates our study among previous experiments that compared horizontal and vertical surfaces. We note that in most cases, display orientation was not the only or main factor of interest. (A second study presented in
[17] examined the effects of the arrangement of users around a horizontal display, which is also relevant to our findings.) In some of these studies, orientation was confounded with other factors (e.g., sitting versus standing [2, 17], digital versus nondigital [18]). In another related study, only a single pen was used [2], thus perhaps making it easier to share in the horizontal condition where the pen can be placed in the center of the table. Our study eliminates these confounds by having participants stand in both conditions and by allowing participants to write simultaneously with multiple pens. Table 1 also demonstrates that our study is consistent with previous work in terms of group size and number of groups studied.

The studies summarized in Table 1 have a wide variation in tasks, input, data, group size, and so forth. This makes it difficult to draw overall conclusions about the advantages and disadvantages of different orientations and to predict which would best encourage participation in a pair-based design task. Nonetheless, in the remainder of this section, we identify commonalities among these findings, which we then use to motivate our hypotheses in the next section.

One fairly well-supported finding is that more equal physical interaction takes place with tabletop interfaces. Studies have reported that asymmetrical roles tend to develop with vertical displays, whereby one user becomes the primary interactor or "scribe" [2]. Difficulty in switching control of the display is likely the largest factor in systems where control must be explicitly passed from one user to another $[2,18]$. For example, in a single-pen system, the need to explicitly pass a pen with a vertical display (rather than placing it in a central location on a horizontal display) was observed to reduce people switching control of the display [2]. In addition, seated participants may be reluctant to get up and walk to a distant display. Multitouch tables have been found to encourage equitable physical interaction $[20,21]$ and more physical gestures [2, 17]. Our study extends earlier results by examining whether greater equality of physical participation on horizontal surfaces still holds true when control does not need to be explicitly passed, and when all users are standing, so they do not need to get up in order to interact.

A greater amount of discussion has also been reported with horizontal displays as compared to vertical $[2,17]$ displays. This can likely be attributed to the fact that adults generally prefer holding conversations face to face or in corner seating arrangements [3].

Equal participation of all users in a collaborative task is often desirable, since a dominant individual may stifle discussion, generation of ideas, and sharing of relevant information [20]. Thus, a display orientation that encourages equal verbal participation may be advantageous. However, Marshall et al. [20] and Rogers et al. [21] both found that display orientation had little influence on this outcome. Although multitouch tabletop displays led to more equitable physical interaction, this equality did not extend to verbal participation. Dominant users continued to dominate the conversation, while quiet users continued to be quiet.

We have heard informal claims that horizontal displays support better face-to-face contact among small groups, but experimental evidence on this topic is quite sparse. Some 
TABLE 1: Summary of horizontal and vertical display orientation studies (H: horizontal, V: vertical).

\begin{tabular}{|c|c|c|c|c|c|c|c|c|}
\hline Study & $\begin{array}{c}\text { Digital (D) or } \\
\text { nondigital (ND) }\end{array}$ & Input & Task & $\begin{array}{c}\text { Orientation- } \\
\text { dependent } \\
\text { data? }\end{array}$ & $\begin{array}{c}\text { Artifacts } \\
\text { created or } \\
\text { used? }\end{array}$ & $\begin{array}{l}\text { Sit or } \\
\text { stand }\end{array}$ & Group size & $\begin{array}{l}\text { Number of } \\
\text { groups }\end{array}$ \\
\hline RL04 [2] & $\mathrm{D}$ & Single pen & $\begin{array}{l}\text { Travel itinerary } \\
\text { planning }\end{array}$ & Yes & Both & $\begin{array}{c}\mathrm{H}-\text { sit } \\
\text { V-sit } \\
\text { except } \\
\text { interactor }\end{array}$ & 3 & 8 \\
\hline IHK $* 05$ [17] & ND & Pens & Route planning & No & Used & $\begin{array}{c}\mathrm{H}-\text { sit } \\
\mathrm{V}-\text { stand }\end{array}$ & 2 & 6 \\
\hline PRR09 [18] & $\begin{array}{c}1 \mathrm{D}(\mathrm{H}), 3 \mathrm{ND} \\
(\mathrm{V}, \text { other })\end{array}$ & $\begin{array}{c}\text { ND-pens } \\
\text { D-multitouch }\end{array}$ & $\begin{array}{l}\text { Concept } \\
\text { mapping }\end{array}$ & Yes & Created & Free form & $9-10$ & $\begin{array}{l}\text { 4-one per } \\
\text { condition }\end{array}$ \\
\hline PS08 [19] & $\mathrm{D}$ & $\begin{array}{l}\text { Multiple mice, } \\
\text { laser pointers }\end{array}$ & $\begin{array}{c}\text { Target } \\
\text { acquisition }\end{array}$ & No & Used & Free form & $1,2,3$ & 12 \\
\hline Our study & ND & Pens & Software design & Yes & Created & Stand & 2 & 10 \\
\hline
\end{tabular}

evidence comes from Lindley and Monk [22]: participants seated in a face-to-face arrangement around a desktop computer gazed at each other more often than those seated in a presenter/audience arrangement. Similarly, Inkpen et al. [17] found that participants seated at right angles at a horizontal display looked at each other significantly more often than when seated side by side or across from each other. However, they did not find a significant difference between horizontal and vertical displays when participants were arranged side by side. The fixed seating arrangements and the difference of sitting at the horizontal display versus standing at the vertical display may have influenced these results. In contrast, our study avoids a sit/stand confound and does not constrain participant arrangements around the horizontal display.

Though less relevant to our work, it is worth mentioning that horizontal and vertical displays have also been compared for individual work tasks. For example, Morris et al. [23] compared horizontal and vertical displays (as well as paper and tablets) for a reading and summarization task, finding that no single display was best for all parts of the task. For instance, the vertical displays were strongly preferred for writing, but strongly disliked for annotation. In a field study of horizontal and vertical displays in personal office environments, Morris et al. [24] found that the horizontal display was used less than the vertical one and was used in a more peripheral way. Both of these studies identified ergonomic problems of horizontal displays and suggested that for individual work it should be possible to angle a horizontal display like a drafting table. Müller-Tomfelde et al. [25] found a similar result: most participants in their study preferred interacting with a tilted workspace over a fully horizontal workspace during a distributed collaboration task. Note that in this situation, only one user was interacting with each display, so it is not clear whether these results would extend to collaboration on a shared display.

\section{Objective and Hypotheses}

We aimed to answer the question, "How does a horizontal surface differ from a vertical surface when used by dyads for a constructive design task?" We chose to focus on a design task because people commonly collaborate around shared displays when brainstorming and refining design ideas. By constructive design, we mean design that involves actively creating a visual representation of the design, as compared to modifying an existing representation or working abstractly without any visual representation. We also focus on data that has an orientation, again for external validity, as most design information has a preferred viewing orientation.

Our study tested the following hypotheses:

(H1) Greater Face-to-Face Contact (i.e., More Glances at a Partner's Face) with Horizontal. Since users can arrange themselves face to face around a horizontal display, they will look at each other more often.

(H2) More Discussion with Horizontal. Horizontal displays will encourage more discussion due to the face-toface arrangement, as found in related studies.

(H3) Equality of Participation in Discussions will not Differ Between Orientations. Prior experiments tested the conjecture that face-to-face arrangements would encourage more equal verbal participation, but found this to be untrue $[20,21]$.

(H4) Increased Equality of Physical Interactions with Horizontal. Physical accessibility of the tabletop surface may encourage equal interaction. Vertical displays may encourage defined interactor/audience roles, and audience members may be reluctant to approach the surface to take control [2].

\section{Methods}

Pairs of skilled software designers collaboratively designed a UML-based software architecture on both horizontal and vertical whiteboards. We chose this task domain because software designers frequently work in pairs or small groups to design software on whiteboards, and there are several current research efforts to design electronic whiteboards for UML diagram design. The task was also a challenging design problem involving knowledge creation often seen in 
the real world. To avoid constraining participant activities based on a particular software tool, input technology, or software controls, participants used nondigital whiteboards and standard whiteboard markers. Although this makes the task a noncomputerized one, we felt that this approach offered the greatest flexibility to users so that the resulting natural behaviours could be used to inform the design of digital whiteboard systems. There is an established history in computer-supported cooperative work in studying nondigital settings prior to designing digital support technologies (e.g., $[16,17])$. Dyads were chosen over slightly larger groups for simplicity in terms of recruitment and analysis, and because most interactions with whiteboards involve groups of 2 to 3 people [26]; however, future work should examine larger groups since group size is known to influence group dynamics [27].

4.1. Participants. Ten pairs of Computer Scientists participated in the study ( 5 pairs of students from a university setting and 5 pairs of software professionals from industry). All 20 participants ( 15 males and 5 females) were experienced developers. Industry professionals were mostly entry-level software developers, but this group also included a team lead, a senior software architect, and a product analyst. All participants had previous knowledge of UML, but their practical experience with it varied from a little use in past university courses to regular use on most software projects. Participants ranged in age from 22 to $51(M=32.4, S D=$ 8.5). Participants had either normal or corrected vision. Each participant was compensated with a \$20 gift certificate.

4.2. Apparatus. As shown in Figure 1, participants sketched UML diagrams on two $48^{\prime \prime} \times 36^{\prime \prime}$ whiteboards: one mounted to a wall in landscape format, with its bottom edge $39^{\prime \prime}$ from the ground, and the other on a tabletop $35^{\prime \prime}$ from the ground. Each participant was given a dry erase marker (one red, one blue) and an eraser brush. Participants stood around the whiteboard in both vertical and horizontal conditions. We focused on standing configurations to eliminate any sit/stand confound between display orientations, to ensure that participants could move around freely, and because people often stand around vertical whiteboards when brainstorming UML software designs.

Video footage captured participants' interactions on the whiteboard and with each other. Two cameras were used: for the vertical condition, one camera was placed behind the participants and one off to the side; for the horizontal condition, cameras were placed on opposite sides, above and away from the table. A still image of each whiteboard was taken at the end of each session.

4.3. Tasks. Each pair was instructed to design two software systems using standard UML class diagrams. They were given a requirement document containing functional system requirements, recommended classes, and use case diagrams. The two tasks were chosen to be similar in complexity (as evaluated by an experienced software developer). Task 1 was a restaurant order management system and Task 2 was a hospital patient management system.

4.4. Procedure. Each session took approximately 60 minutes. Participants completed the following pretrial, trial, and posttrial activities.

(i) Pretrial. Participants were briefed, signed a consent form, and filled out a pretrial questionnaire that contained demographic questions, questions about previous UML experience, and (for industry participants) questions about their use of whiteboards at work and their current job responsibilities. The briefing included a 5-minute refresher summary of UML syntax.

(ii) Trial. Participants started with Task 1 in one display configuration, were offered a 5-minute break, and then performed Task 2 in the other configuration. Horizontal and vertical configurations were counter-balanced for order. Pairs were given up to 20 minutes for each task.

(iii) Posttrial. The session concluded with a posttrial questionnaire that asked questions about which task and surface each participant preferred.

4.5. Measures. We primarily measured counts and durations of activities, determined through manual video coding. Specific activities that we coded are described in the results section.

One of our goals was to measure the relative participation (verbal and physical) by the two individuals. To measure this, we use an index of inequality, $I$, previously used by Marshall et al. [20] I varies from zero (perfect equality; all participants contribute equally) to one (perfect inequality; only one participant contributes). $I$ is robust to small numbers of participants and is normalized for the number of participants per group, enabling comparison across studies with different group sizes. It was calculated for each group and condition using (1), where $N$ is the group size; $E_{i}$ is the expected cumulative proportion of events if each participant contributes equally; and $O_{i}$ is the observed cumulative proportion of events, starting with the participant who contributed least:

$$
I=\frac{(1 / N) \sum_{i=1}^{N}\left(E_{i}-O_{i}\right)}{(1 / 2)(1-1 / N)},
$$

when $N=2, I=2\left(0.5-O_{1}\right)$, where $O_{1}$ is the observed proportion for the participant who contributed less.

\section{Results}

We organize our results according to the four hypotheses. We also report user preferences for orientation and task. Q-Q plots suggested that all data were normally distributed. When Mauchly's test of sphericity indicated that it was necessary, we used a Huynh-Feldt correction for repeated measures ANOVA. 


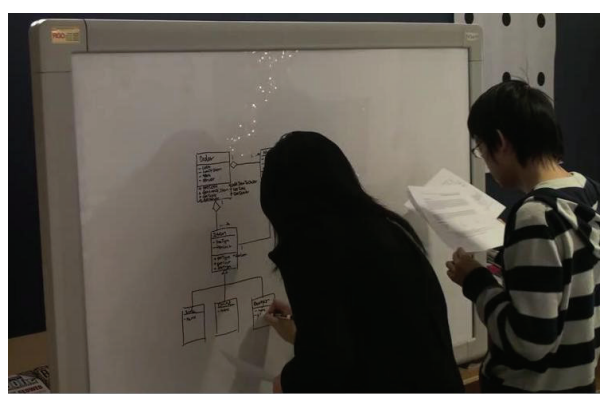

(a) Vertical configuration

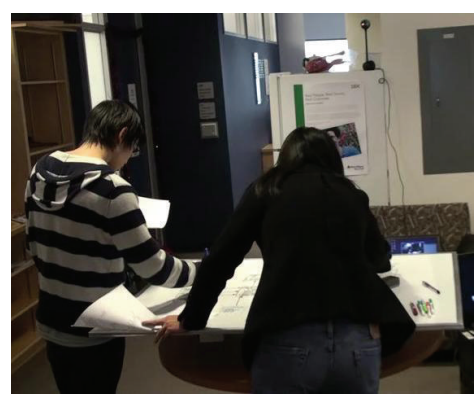

(b) Horizontal configuration

FIgURe 1: Participants working in vertical and horizontal configurations.

For horizontal displays, some of our results presumably depend on the arrangement of users around the table. We classified the arrangements as side-by-side, opposite one another, or kitty-corner (facing perpendicular directions). Total time spent in each configuration was video coded. Sideby-side and kitty-corner were both substantially used, but opposite was used only rarely. Only two groups remained side-by-side $100 \%$ of the time. Average percent time in each configuration was side-by-side $M=53.6 \%$, opposite, $M=$ $7.7 \%$, and kitty corner, $M=38.6 \%$.

For each hypothesis, we first examined the overall results comparing horizontal to vertical and then broke down the horizontal results by arrangement. The opposite arrangement was excluded due to its rare use. For the arrangement analysis, we adjusted for the different amounts of time spent in each arrangement by calculating results on a per-minute basis.

5.1. H1: Face-to-Face Contact. H1 stated that participants would glance at each other's faces more often in the horizontal condition. To investigate this, we coded (from video) all of the times when each participant looked at the other's face. Since many of the looks consisted of a quick glance that would be difficult to time, we counted the looks rather than attempting to code their duration.

Figure 2 shows the unexpected result that participants looked at each other's faces significantly more often in the vertical orientation than the horizontal orientation, $t(19)=$ 3.2, $p=0.005$.

The number of looks per minute was higher for vertical than for both horizontal arrangements (side-by-side and kitty corner), as shown in Figure 3. Repeated measures ANOVA revealed a significant difference $(F(2,38)=9.8, p<$ $\left.0.001, \eta_{p}^{2}=0.34\right)$. Bonferroni-corrected post hoc tests showed that horizontal side-by-side was significantly different from vertical $(p=0.001)$.

5.2. H2: Total Discussion. $\mathrm{H} 2$ stated that there would be more overall discussion in the horizontal condition. We coded the duration of verbal utterances from each participant and summed these for each condition to examine the total overall amount of conversation. Mean values for horizontal and

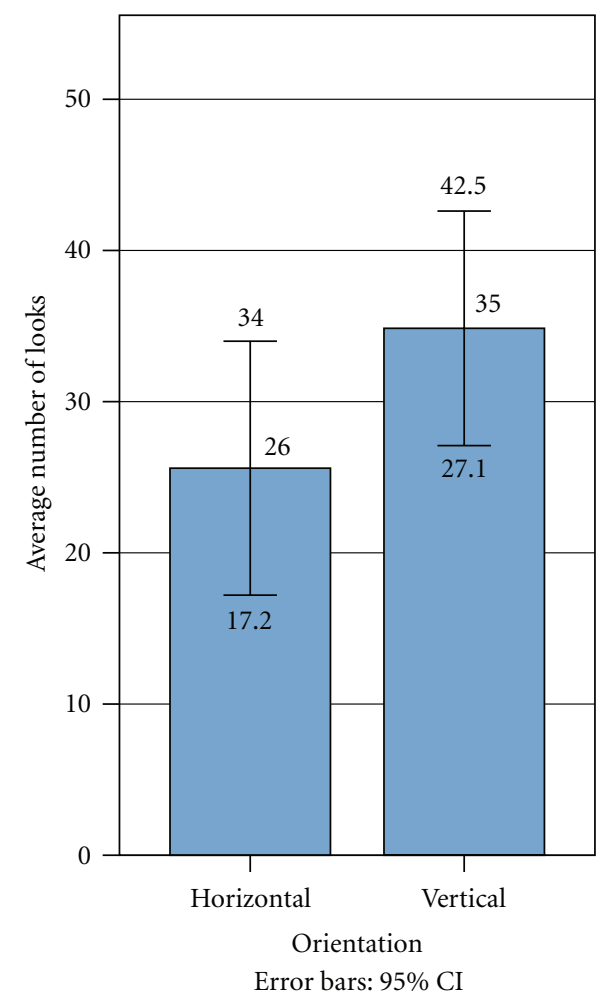

FIgURE 2: Average number of looks (per person).

vertical orientations were very similar (see Figure 4). To gain a better understanding of this similarity, we calculated differences from the mean as shown in the boxplots of Figure 5. The similarity suggests that horizontal and vertical are likely equivalent, though more participants would be needed to test this statistically.

With the horizontal display, time talking varied with the user arrangement (see Figure 6). Perhaps surprisingly, there was more talking per minute when side-by-side as compared to kitty-corner, and this was also higher than sideby-side with a vertical display. Repeated measures ANOVA showed an overall significant effect $(F(1.3,24.9)=20.2, p<$ $\left.0.001, \eta_{p}^{2}=0.52\right)$. Bonferroni-corrected post hoc tests showed that all pairs were significantly different $(p<0.008)$. 


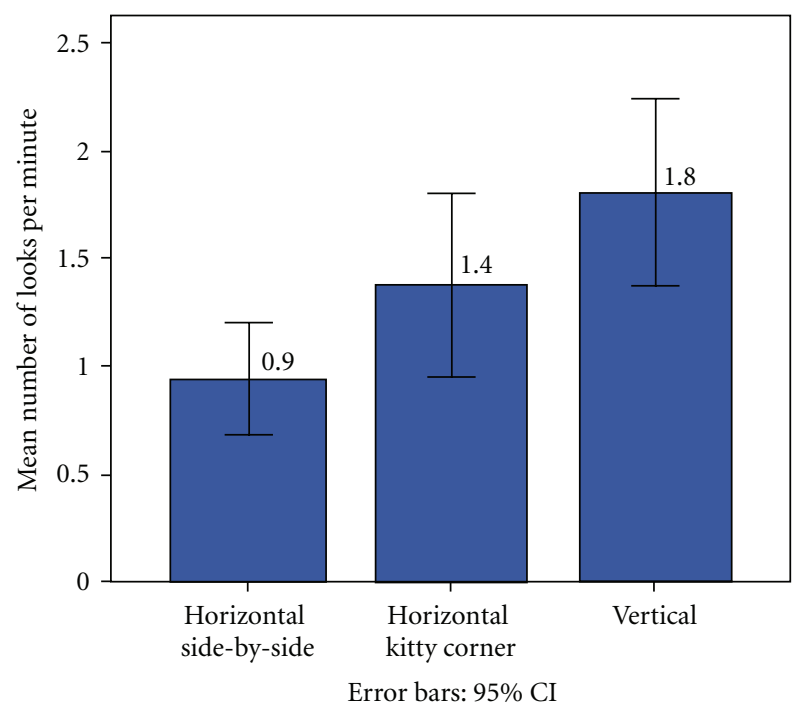

FIGURE 3: Average looks per minute (per person).

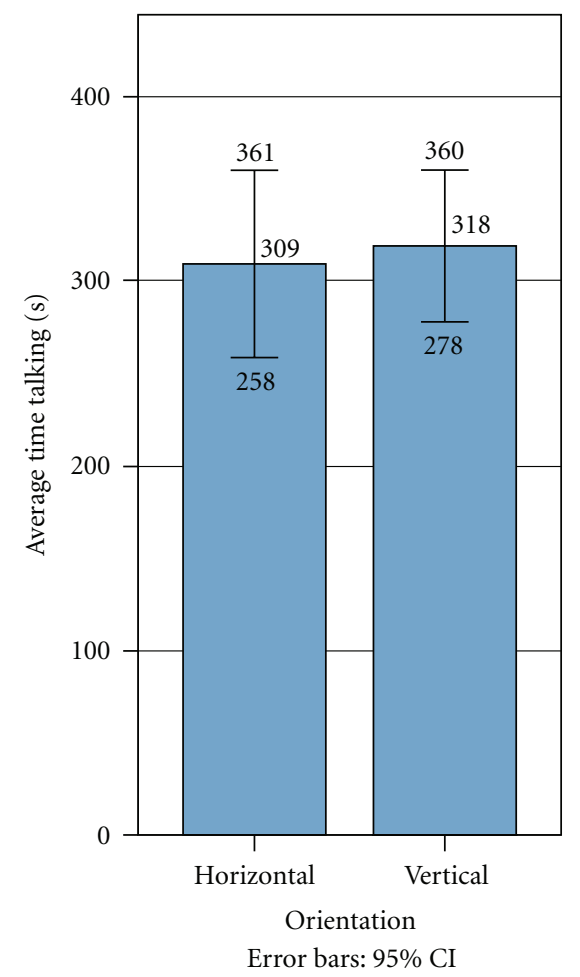

FIgURE 4: Average time talking (per person).

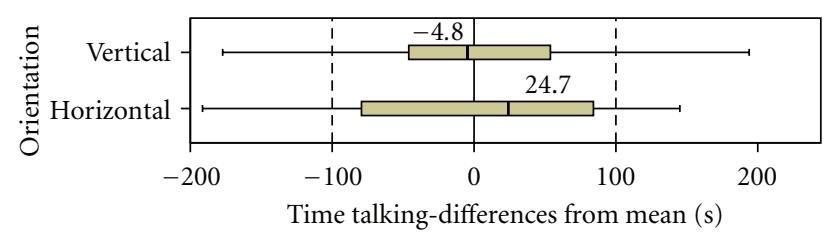

FIGURE 5: Equivalence of talking times, shown as differences from the mean.

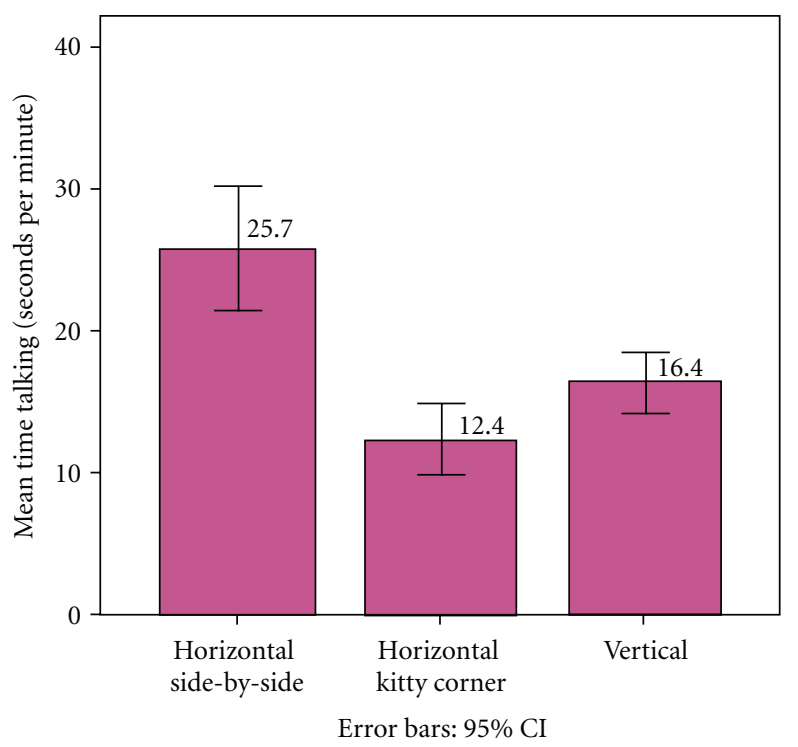

FIGURE 6: Average time talking per minute (per person).

5.3. H3: Equality of Verbal Participation. Prior evidence [20, 21] indicated that relative contributions of each participant to discussion would not be influenced by the orientation. To examine this, we first calculated the proportion of speaking time by the less talkative person in each condition. We used this value to calculate the index of inequality. Figure $7(\mathrm{a})$ shows these data. Vertical had slightly greater equality (lower value), but the difference was not statistically significant. With the horizontal display, there was greater equality with side-by-side $(M=0.22)$ than with kitty corner $(M=0.34)$, but this difference was also not significant.

5.4. H4: Equality of Physical Participation. H4 stated that participants' physical interactions would be more equal in the horizontal condition. To examine this, we coded the durations of drawing and erasing and calculated the proportion of interactions done by the person who interacted the least. We then used this proportion to calculate the index of inequality. Figure 7(b) shows that equality was slightly greater with the horizontal orientation, though this difference was not statistically significant. Inequality of physical participation also did not vary significantly with horizontal arrangement (side-by-side versus kitty corner).

5.5. Orientation and Task Preference. Preference for the conditions was equally split: 10 participants preferred horizontal surface orientation and 10 preferred vertical surface orientation. This echoes results found elsewhere [17] for preference of horizontal and vertical surfaces. Preference for the two tasks was close to equally split (8 preferred task 1,11 preferred task 2 , and 1 had no preference). There was a strong correspondence between the preferred task and preferred orientation ( $75 \%$ agreement), so it is unclear which is the most important factor influencing preference.

Analysis of the poststudy questionnaire results gives some further insight. Regarding the display conditions, 


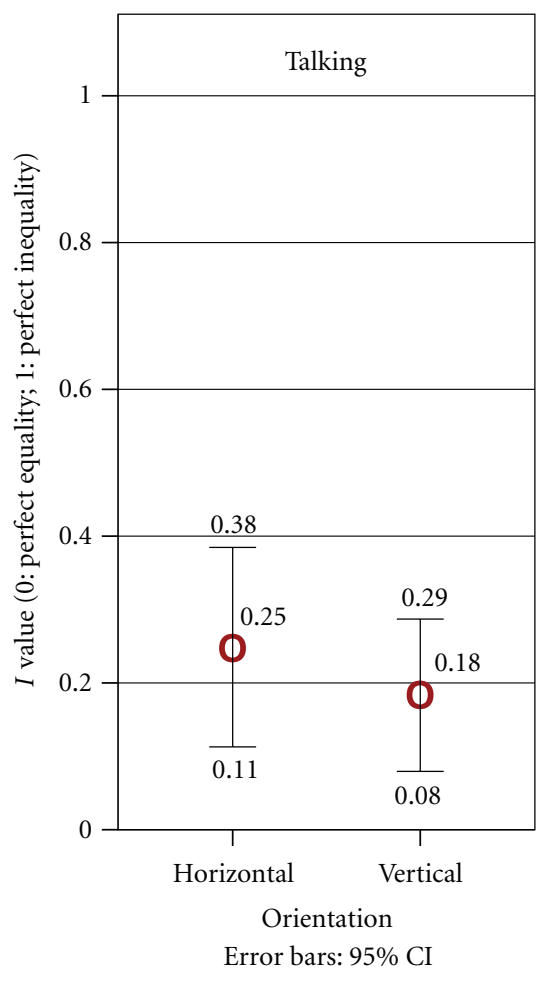

(a)

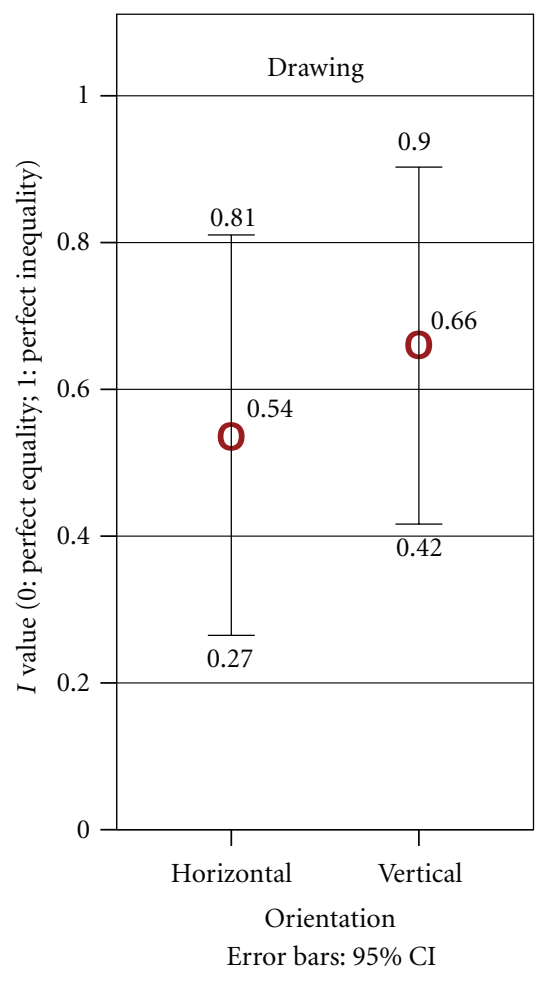

(b)

Figure 7: Equality of (a) talking and (b) drawing/erasing.

most participants referred to ergonomics: they preferred the display that they felt was easiest on their head and neck position, or that facilitated writing or drawing. However, which display did this best was not consistent between participants. For example, one participant who preferred horizontal wrote, "I'm short! lol. I thought it was easier to stand with someone and communicate by pointing." whereas one who preferred vertical wrote, "I'm short, it's easier to access the wall mounted board". Regarding the tasks, we found that the two tasks were not perfectly equivalent. The restaurant scenario was conceptually simpler for many participants. Some preferred this simplicity while others preferred a greater challenge. Others expressed a preference based on which topic interested them the most. All participants gave separate reasons for their preference of task and display, suggesting that the correlation may be accidental, or that if the issues are conflated, it is at a subconscious level (e.g., it is possible that a more comfortable working position influenced them to subconsciously like the task more, or vice versa).

\section{Discussion}

Our experiment calls into question many assumptions we made regarding advantages of horizontal displays. Most importantly, face-to-face contact was found to be significantly greater with vertical displays, not horizontal (opposite of H1). This occurred despite the fact that participants spent over $46 \%$ of their time standing in face-to-face configurations with the horizontal condition. We had expected these face-to-face arrangements to enable more glances between participants. However, our observations and poststudy questionnaire responses suggest that looking at the other person was more cumbersome in the horizontal condition. With vertical, the board and the other person's head were at a similar height, so looking at the person required only turning one's head horizontally. With the horizontal board, however, looking at the other person typically involved turning one's head horizontally as well as looking up. This suggests that when users are standing, faceto-face contact may be easier with vertical displays. Results may of course differ for seated configurations. Height of the table may also have an impact; a higher table might make face-to-face contact easier but would probably also make writing more difficult. As expected, there were a greater number of looks with the horizontal condition when users stood in a kitty corner arrangement as compared to side-byside. This reaffirms earlier results [17]. Both were still less than the vertical condition, however.

These results demonstrate that horizontal displays alone do not cause more face-to-face contact, contradicting a common assumption about interactive surfaces. Thus a horizontal display should not be chosen purely for this reason, and other factors need to be taken into consideration. In fact, at least if users will be standing, face to face contact may be easier with a vertical display, which also has the added benefit of all users seeing the same perspective. This result might be counterintuitive to some designers. 
Our results did not support H2. Horizontal and vertical may be equivalent overall in terms of total discussion time; the strong similarity suggests that it may be worthwhile to conduct a more rigorous test of equivalence with more participants. It was interesting to see that significantly more discussion took place when users were side-by-side with the horizontal board, as compared to kitty corner or side-byside with the vertical board. Rodden et al.'s [28] findings for trade show that displays might explain this; they found that side-by-side arrangements could ease social awkwardness and encourage shy people to talk. It is also possible that users moved to a side-by-side arrangement when talking, to gain a common perspective. However, our observations suggest that changes in arrangement were usually motivated by writing: participants moved in order to write somewhere that was hard to reach, or moved out of the way so the other person could write.

Our study did not show evidence of a significant difference between horizontal and vertical orientation for equality of physical or verbal participation ( $\mathrm{H} 3$ was supported but H4 was not supported). Additional participants may reveal a significant difference, but it appears to be small.

We do note that our evaluations of discussion looked only at overall quantity; we did not consider the formality of the discussion nor the quality of ideas generated. Although there was no observable quality difference in the final design solutions, there is still some possibility of differences in the quality of discussion between horizontal and vertical configurations. A qualitative analysis could examine the discussion in this way, as well as other differences such as the way in which people collaborated; however, this was beyond the scope of the current work. We did qualitatively examine the resulting UML diagrams, looking for differences such as software design quality, diagram structure, and types and location of strokes placed by each participant (observable through the different coloured pens). However, we found no observable diagram differences between horizontal and vertical conditions.

It is also interesting to note that we did not observe territorial behaviour in our study, unlike reports from previous work [10]. Although participants tended to work in different areas of the whiteboard at any given time, they appeared to have no trouble moving to another area or adding onto a part of the diagram created by their partner. We do not interpret this to mean that territoriality is not important, however. We suspect that the UML diagram was simply treated as a shared representation so that no ownership was implied by creation of its various parts.

Our results must be considered in the context of the choices we made in our experimental setup. We focused on dyads, standing configurations, and a design task with naturally oriented information. Some of the significant differences seen in previous studies that were not seen in our study (despite a similar number of participants) may be attributable to some of these factors. For instance, equality of physical interaction may depend less on the orientation and more on all users being able to easily reach the surface and use it without explicitly taking control from another user. Ergonomics of being able to view the other person around the tabletop may have been easier in a seated configuration, but this would have introduced a confounding factor and constrained positioning around the display. Additionally, in our study, the orientation of the information may have biased participants to spend more time in a side-by-side arrangement than might otherwise be seen with horizontal displays, though it was interesting that more discussion actually took place in the side-by-side arrangement. Results for digital UML diagrams may differ slightly from our whiteboard-based results since digital UML components can be more easily rotated or moved. However, we suspect that movement would be used mainly to make space for new information and that actions that disrupt the global view (e.g., rotation and movement for the purpose of obtaining a better view) would happen rarely since they would impact the other participant. Finally, it is unclear to what extent the UML design task is similar to other types of design tasks. While we expect that many other design tasks would have similar coupling, division of labour, and coordination characteristics, some of our results may be particular to the software engineering domain that we studied.

Our results should not be taken to mean that one orientation is necessarily better than another, but that the choice of orientation may depend on the task at hand as well as personal preference. It is useful to note that there were fewer differences between horizontal and vertical surfaces than expected; for example, there was no observable difference in the quality of the resulting design work. People were able to adapt, and they found effective ways to work in both configurations. Perhaps the choice of horizontal versus vertical displays is not as important as we might have thought. This finding would be very beneficial to organizations with limited resources, since vertical displays are more commonplace, require less floor space, and are often less expensive than horizontal ones. Or perhaps other factors such as input and physical positioning (e.g., sitting versus standing) have a larger impact on group dynamics than the surface orientation.

\section{Conclusion and Future Work}

Our results add new empirical evidence regarding how display orientation impacts collaboration. Perhaps our most interesting finding is that looking at a collaborator's face can be awkward when standing at a horizontal display and occurs less often than at a vertical display; vertical displays may be better for standing users in terms of face-to-face contact. In contrast, the most discussion took place when participants stood side-by-side next to a horizontal display. We found no evidence for a difference in equality of physical or verbal participation for horizontal versus vertical conditions, in contrast to some earlier studies. In summary, our findings demonstrate that dyads can work effectively with both vertical and horizontal surfaces. What was most surprising to us was that we did not observe a strong motivation to prefer one orientation over the other. We feel that we need to follow this up with future work, where we would like to investigate the relationships between physical positioning and display 
orientation by constraining participant's spatial movement and comparing sitting versus standing positions. We would also like to consider tilted displays as a compromise between horizontal and vertical. Additional conclusive findings will help future designers of whiteboard applications that support small groups performing design tasks on both horizontal and vertical displays.

\section{Acknowledgment}

This work was supported by the Natural Sciences and Engineering Research Council of Canada (NSERC).

\section{References}

[1] P. Marshall, Y. Rogers, and E. Hornecker, "Are tangible interfaces really any better than other kinds of interfaces?" in Proceedings of the Tangible User Interfaces in Context and Theory Workshop (CHI'07), 2007.

[2] Y. Rogers and S. Lindley, "Collaborating around vertical and horizontal large interactive displays: which way is best?" Interacting with Computers, vol. 16, no. 6, pp. 1133-1152, 2004.

[3] S. D. Scott, K. D. Grant, and R. L. Mandryk, "System guidelines for co-located, collaborative work on a tabletop display," in Proceedings of the European Conference Computer-Supported Cooperative Work, pp. 159-178, 2003.

[4] U. Dekel and J. D. Herbsleb, "Notation and representation in collaborative object-oriented design: an observational study," in Proceedings of the ACM SIGPLAN Conference on ObjectOriented Programming, Systems, Languages, and Applications (OOPSLA '07), pp. 261-280, October 2007.

[5] Q. Chen, J. Grundy, and J. Hosking, "An e-whiteboard application to support early design-stage sketching of UML diagrams," in Proceedings of the IEEE Symposium Human Centric Computing Languages and Environments (HCC '03), pp. 219-226, 2003.

[6] C. H. Damm, K. M. Hansen, and M. Thomsen, "Tool support for cooperative object-oriented design: gesture based modeling on an electronic whiteboard," in Proceedings of the SIGCHI Conference on Human Factors in Computing Systems (CHI '00), pp. 518-525, April 2000.

[7] J. Wu and T. C. N. Graham, "The software design board: a tool supporting workstyle transitions in collaborative software design," in Proceedings of the Engineering Human Computer Interaction and Interactive Systems, vol. 3425 of Lecture Notes in Computer Science, pp. 363-382, 2005.

[8] A. Tang, M. Tory, B. Po, P. Neumann, and S. Carpendale, "Collaborative coupling over tabletop displays," in Proceedings of the ACM Conference on Human Factors in Computing Systems (CHI '06), pp. 1181-1190, April 2006.

[9] O. Kulyk and G. van der Veer, "Situational awareness support to enhance teamwork in collaborative environments," in Proceedings of the 15th European conference on Cognitive Ergonomics, pp. 1-5, 2008.

[10] S. D. Scott, S. M. T. Carpendale, and K. M. Inkpen, "Territoriality in collaborative tabletop workspaces," in Proceedings of the ACM Conference on Computer Supported Cooperative Work (CSCW'04), pp. 294-303, November 2004.

[11] K. Hawkey, M. Kellar, D. Reilly, T. Whalen, K. M. Inkpen et al., "The proximity factor: impact of distance on colocated collaboration," in Proceedings of the International ACM
SIGGROUP Conference on Supporting Group Work (GROUP '05), pp. 31-40, November 2005.

[12] S. Bachl, M. Tomitsch, K. Kappel, and T. Grechenig, "The effects of personal displays and transfer techniques on collaboration strategies in multi-touch based multi-display environments," in Proceedings of the 13 International Conference on Human-Computer Interaction (INTERACT '11), vol. 6948 of Lecture Notes in Computer Science, pp. 373-390, 2011.

[13] M. Haller, J. Leitner, T. Seifried et al., "The NiCE discussion room: integrating paper and digital media to support colocated group meetings ," in Proceedings of the 28th Annual CHI Conference on Human Factors in Computing Systems (CHI '10), pp. 609-618, April 2010.

[14] J. R. Wallace, S. D. Scott, T. Stutz, T. Enns, and K. Inkpen, "Investigating teamwork and taskwork in single- and multidisplay groupware systems," Personal and Ubiquitous Computing, vol. 13, no. 8, pp. 569-581, 2009.

[15] J. C. Tang, "Findings from observational studies of collaborative work," International Journal of Man-Machine Studies, vol. 34, no. 2, pp. 143-160, 1991.

[16] A. Tang, J. Lanir, S. Greenberg, and S. Fels, "Supporting transitions in work: informing large display application design by understanding whiteboard use," in Proceedings of the ACM SIGCHI International Conference on Supporting Group Work (GROUP '09), pp. 149-158, May 2009.

[17] K. Inkpen, K. Hawkey, M. Kellar et al., "Exploring display factors that influence co-located collaboration: angle, size, number, and user arrangement," in Proceedings of the HumanComputer Interaction Conference (HCI '05), 2005.

[18] N. Pantidi, Y. Rogers, and H. Robinson, "Is the writing on the wall for tabletops?" in Proceedings of the 12th International Conference on Human-Computer Interaction: Part II, vol. 5727 of Lecture Notes in Computer Science, pp. 125-137, 2009.

[19] A. Pavlovych and W. Stuerzlinger, "Effect of screen configuration and interaction devices in shared display groupware," in Proceedings of the 3rd ACM International Workshop on HumanCentered Computing (HCC '08), pp. 49-56, October 2008.

[20] P. Marshall, E. Hornecker, R. Morris, N. S. Dalton, and Y. Rogers, "When the fingers do the talking: a study of group participation with varying constraints to a tabletop interface," in Proceedings of the IEEE Tabletops and Interactive Surfaces (TABLETOP '08), pp. 37-44, October 2008.

[21] Y. Rogers, Y. Lim, W. R. Hazlewood, and P. Marshall, "Equal opportunities: do shareable interfaces promote more group participation than single user displays?" Human-Computer Interaction, vol. 24, no. 1-2, pp. 79-116, 2009.

[22] S. Lindley and A. F. Monk, "Social enjoyment with electronic photograph displays: awareness and control," International Journal of Human Computer Studies, vol. 66, no. 8, pp. 587604, 2008.

[23] M. R. Morris, A. J. B. Brush, and B. R. Meyers, "Reading revisited: evaluating the usability of digital display surfaces for active reading tasks," in Proceedings of the Horizontal Interactive Human-Computer Systems (Tabletop '07), pp. 7986, October 2007.

[24] M. R. Morris, A. J. B. Brush, and B. R. Meyers, "A field study of knowledge workers' use of interactive horizontal displays," in Proceedings of the Horizontal Interactive Human Computer System (TABLETOP '08), pp. 105-112, October 2008.

[25] C. Müller-Tomfelde, A. Wessels, and C. Schremmer, "Tilted tabletops: in between horizontal and vertical workspaces," in Proceedings of the International Workshop on Horizontal Interactive Human Computer System (TABLETOP '08), pp. 4956, October 2008. 
[26] J. Walny, S. Carpendale, N. H. Riche, G. Venolia, and P. Fawcett, "Visual thinking in action: visualizations as used on whiteboards," IEEE Transactions on Visualization and Computer Graphics, vol. 17, no. 12, pp. 2508-2517, 2011.

[27] G. Simmel, "The number of members as determining the sociological form of the group," American Journal of Sociology, vol. 8, pp. 1-46, 1902.

[28] T. Rodden, Y. Rogers, J. Halloran, and I. Taylor, "Designing novel interactional workspaces to support face to face consultations," in Proceedings of the New Horizons Conference on Human Factors in Computing Systems (CHI '03), pp. 57-64, April 2003. 

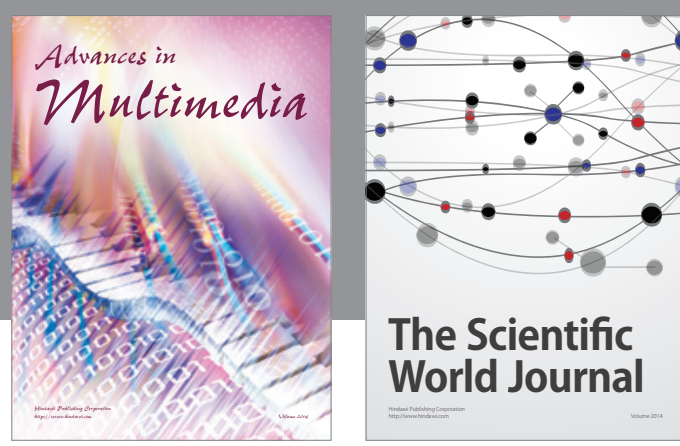

The Scientific World Journal
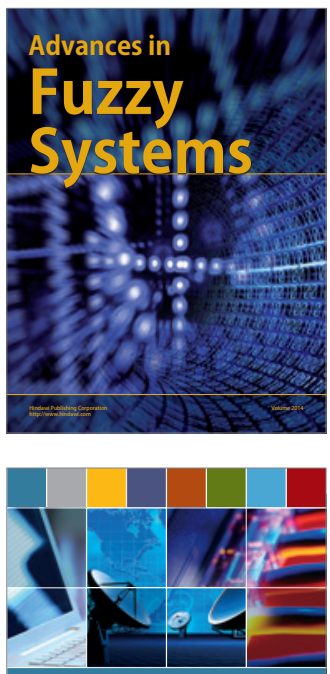

Computer Networks and Communications
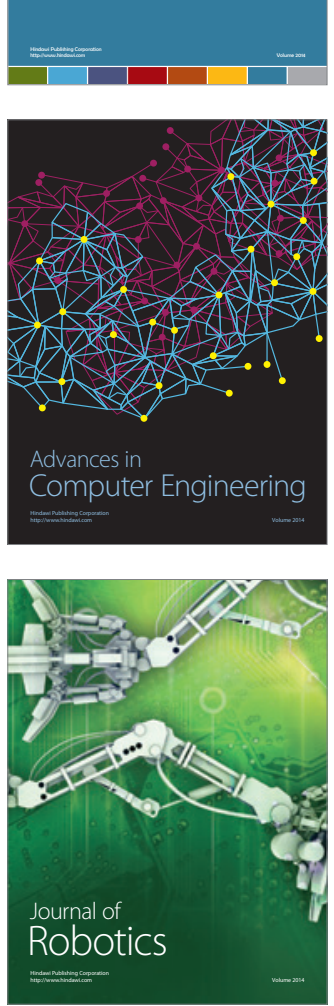
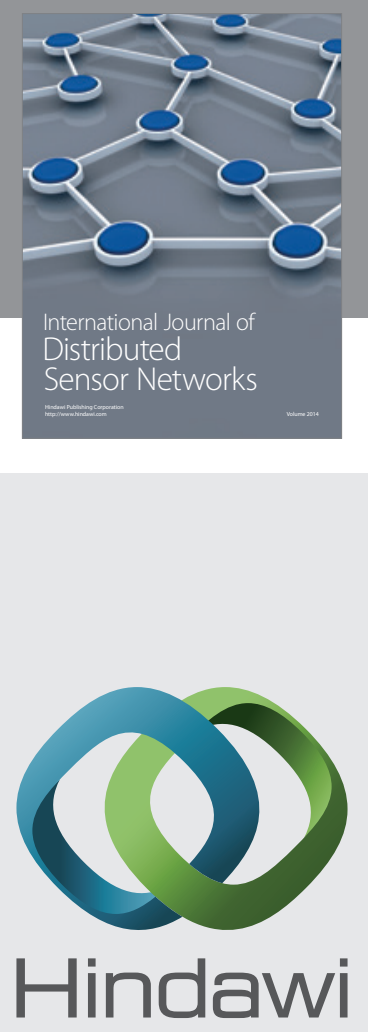

Submit your manuscripts at

http://www.hindawi.com
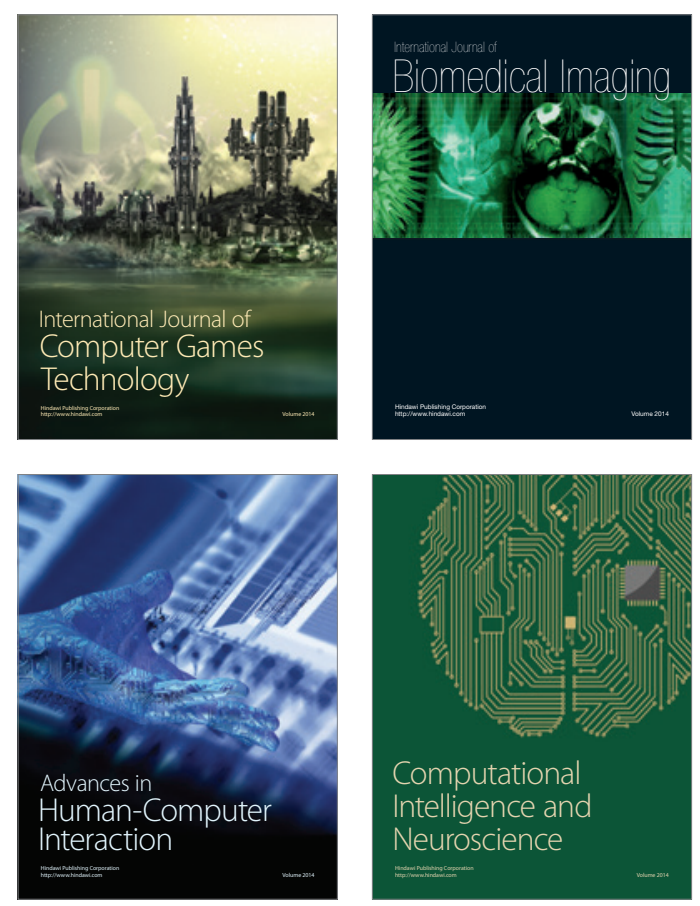
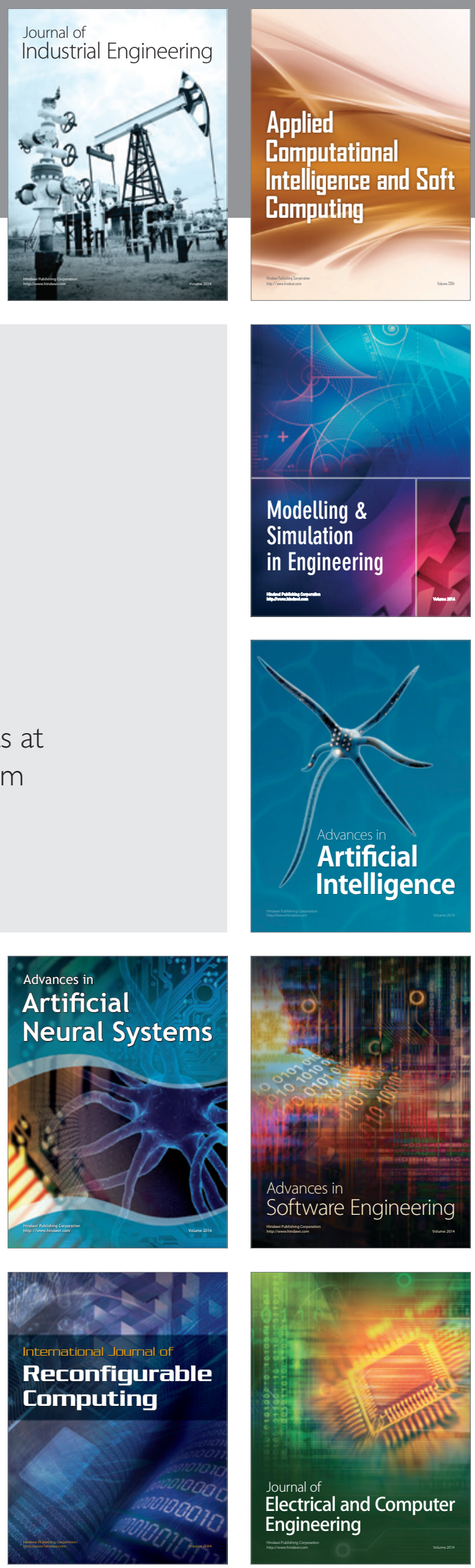